\title{
Improved Dual Closed-loops PWM Control of PM DC Servomotor - a Case Study of Undergraduate Education for Electrical Engineering
}

\author{
Hongtai Cao *
}

\begin{abstract}
PID control method usually has problems of overshoot and oscillation in high order control system, therefore, it is important to improve the control method so as to reduce the overshoot and oscillation. Based on MATLAB simulation, a permanent magnet (PM) DC servomotor control system is studied in this paper. The motor is modeled according to the universal motor theory, and with the help of the fourth order Ronge-Kutta method, its speed control is simulated and compared between two different dual closed-loops PWM control methods. This case study helps undergraduate students to better understand theories related to electrical engineering, such as electrical machinery, power electronics and control theory, as well as digital solution of state equations.
\end{abstract}

Keywords: PM DC motor, Dual closed-loops control, Fourth-order Ronge-Kutta method, Universal motor model theory, PWM

\section{Introduction}

PM DC servomotors play important roles in, for example, transportation, factory manufacture, and many other industries. This is because DC servomotors exhibit wide speed range and excellent speed characteristics. Meanwhile more and more theories are proposed and improved to optimize the control of DC servomotors, while the control strategies are eventually implemented with power electronic converters. Moreover, the control performance can be simply simulated with the systems model which is actually a set of state equations. Therefore, the PM DC servomotor system is an interesting case for undergraduate education, to help the students better understand the control theory, electrical machine theory, power electronics technology, and digital solution of state equations.

Lots of researchers did different researches on DC motor speed control based on computer simulation through different control theories. For example, Pal A.K and Mudi R.K used fuzzy PI controller on speed control of DC motors [1], Zhao D, Li C and Ren J used fuzzy speed control on DC motor speed control [2], and Gupta S K and Varshney P used fractional fuzzy PID controller for speed control of DC motor [3]. Some of the researches obtained desired control results, while others didn't. PID control theory is a classical theory but it has weakness of overshoot and oscillation, thus, fuzzy PID control is developed as a

* College of Electrical Engineering, Zhejiang University, China. (caohongtai2014@gmail.com)

Received 25 September, 2014; Accepted 15 October, 2014 modern PID theory. Many researches obtained excellent results in different motor control with different methods [4].

PWM technology is usually applied in speed control of motors. By way of example, the DC motor speed control using hysteresis controlled synchronized pulse generator [5] is a kind of control method using PWM theory. Compared with fuzzy PID theory, the hysteresis PWM control strategy is simpler and easier to be adopted in the control system.

\section{Universal Motor Model}

There are three main types of motors, DC motors, asynchronous motors and synchronous motors. They have many commons, e.g., they all have stators and rotors, and the interaction between the stator and rotor magnet field produces an electromagnetic torque. Due to these essential commons, a universal motor model theory was established.

\subsection{Hypothesis}

The magnetic circuit is not saturated. This hypothesis will linearize and simplify the expressions of the model. Iterative computations can be applied to linear expressions.

Residual magnetization, eddy current and hysteresis effect in the motor iron cores are negligible. When necessary, residual magnetization can be modeled as a steady excitation current, and the eddy current can be modeled and represented as short-circuited coils. 
Air gap flux density assumes to be of sinusoidal distribution. This will simplify the motor model.

Armature leakage is ignored. Although this will cause some inaccuracy, it will simplify the motor model.

The model of the universal motor is shown in Fig. 1.

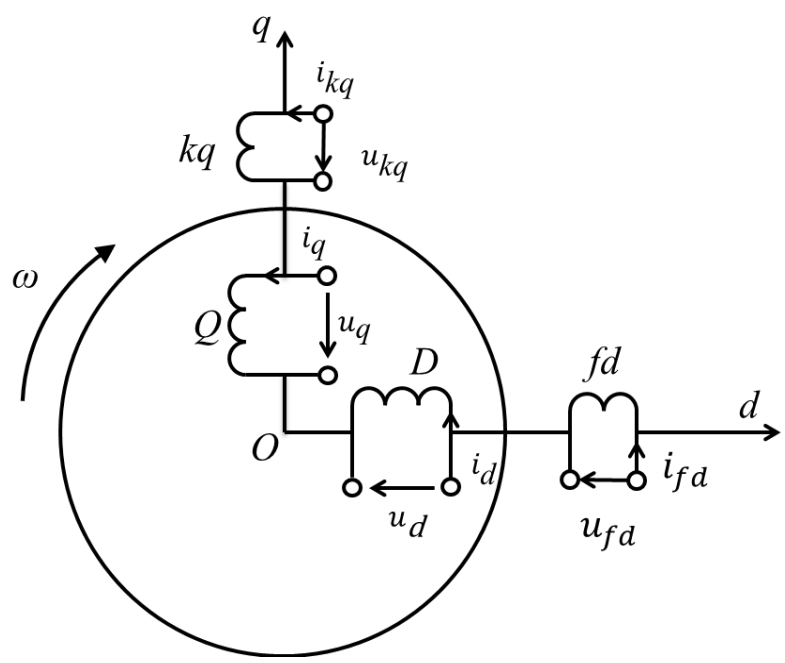

Fig. 1. Universal Motor Model

\subsection{Modeling}

Winding D represents the direct-axis armature winding, and $\mathrm{Q}$ is the quadrature-axis winding. Coil $f d$ represents the direct-axis field winding and $k q$ represents the quadratureaxis field winding. The electromagnetic state equations of the universal motor are:

$$
[U]=\left([R]+[L] \frac{d}{d t}+\omega[G]\right)[I]
$$

where

$$
[U]=\left[\begin{array}{c}
u_{d} \\
u_{q} \\
u_{f d} \\
u_{k q}
\end{array}\right]
$$

represents the voltage of windings,

$$
[R]=\left[\begin{array}{cccc}
R_{d} & 0 & 0 & 0 \\
0 & R_{q} & 0 & 0 \\
0 & 0 & R_{f d} & 0 \\
0 & 0 & 0 & R_{k q}
\end{array}\right]
$$

represents the resistance of windings,

$$
[L]=\left[\begin{array}{cccc}
L_{d} & 0 & M_{a f d} & 0 \\
0 & L_{q} & 0 & M_{a k q} \\
M_{f a d} & 0 & L_{f d} & 0 \\
0 & M_{k a q} & 0 & L_{k q}
\end{array}\right]
$$

represents the inductance of windings,

$$
[G]=\left[\begin{array}{cccc}
0 & -L_{q} & 0 & -M_{a k q} \\
L_{d} & 0 & M_{a f d} & 0 \\
0 & 0 & 0 & 0 \\
0 & 0 & 0 & 0
\end{array}\right]
$$

represents the rotary inductance, and

$$
[I]=\left[\begin{array}{c}
i_{d} \\
i_{q} \\
i_{f d} \\
i_{k q}
\end{array}\right]
$$

represents the current of windings. And, $\omega$ represents the electric angular speed of the rotor.

The mechanical state equation of the universal motor is:

$$
\frac{d}{d t} \Omega=\frac{p\left(\psi_{d} i_{q}-\psi_{q} i_{d}\right)-T_{m}-B \Omega}{J}
$$

where

$T_{m}$ represents the output mechanical torque,

$B$ represents the damping factor,

$J$ represents the rotational inertia,

$p$ represents the number of pole-pairs, and

$\Omega$ represents the mechanical angular speed of the rotor.

For a 2-pole motor, $\Omega=\omega$.

\subsection{State Equations of DC Servomotor}

The PM DC servomotor system is shown in Fig. 2.

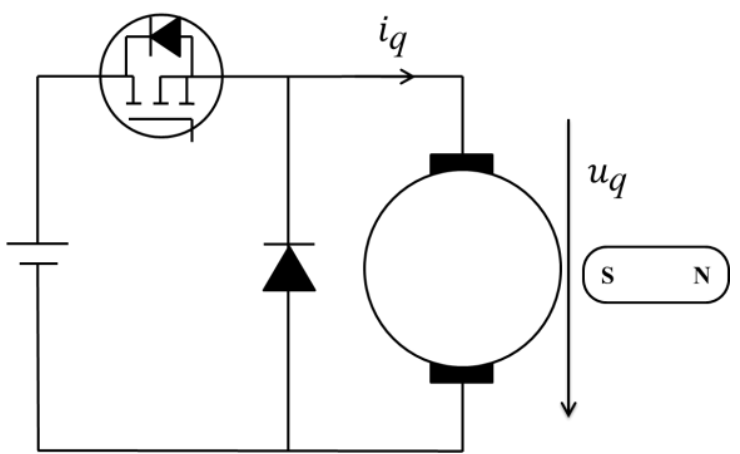

Fig. 2. DC Servomotor System 
The motor itself is simpler than the universal motor. It has one winding only, viz., the armature winding Q. And, its direct-axis field is excited by the magnets only, and the flux linkage is $\Psi_{d}=1 \mathrm{~Wb}$. The armature winding resistance is $R q=0.5 \Omega$, the inductance $\mathrm{Q}$ is $L_{q}=0.05 \mathrm{H}$. The moment of inertia of the rotor is $J=0.002 \mathrm{~kg} \cdot \mathrm{m}^{2}$, and the mechanical damping factor is $B=0.1 \mathrm{~N} \cdot \mathrm{m} /(\mathrm{rad} / \mathrm{s})$. The motor under study has 2 poles. The PM DC servomotor is driven with a buck converter, consisting of an IGBT and a diode which are regarded as ideal devices.

According to the electromagnetic equations and the mechanical equation, the following state equations under no load condition can be obtained:

$$
\begin{gathered}
u_{q}=R_{q} i_{q}+L_{q} \frac{d}{d t} i_{q}+\omega \psi_{d} \\
\frac{d}{d t} \omega=\frac{\psi_{d} i_{q}-B \omega}{J}
\end{gathered}
$$

thus

$$
\begin{gathered}
\frac{d}{d t} i_{q}=\frac{u_{q}-\omega-0.5 i_{q}}{0.05} \\
\frac{d}{d t} \omega=\frac{i_{q}-0.1 \omega}{0.002}
\end{gathered}
$$

Initial conditions are $u_{q}=0, i_{q}=0, \omega=0$. PWM is applied on the buck converter. When PWM $=1$, the IGBT is turned on, thus, $u_{q}=u_{d c}$. When PWM $=0$, the IGBT is turned off, and if $i_{q}>0$, current goes through the free-wheeling diode which is in parallel to the motor armature, thus, $u_{q}=0$.

\subsection{Solution to Differential Equations}

The speed control simulation is based on MATLAB, and the differential equations of (10) and (11) are solved by the fourth-order Ronge-Kutta method. Its steps of the numerical computation are the following.

The differential equation problem format is

$$
\begin{gathered}
\frac{d}{d t}[X]=\mathbf{f}(t,[X]) \\
{[X]_{i+1}=[X]_{i}+h \cdot[X]}
\end{gathered}
$$

where $h$ is step length, and

$$
\begin{gathered}
{[K]_{1}=\mathbf{f}\left(t_{i},[X]_{i}\right)} \\
{[K]_{2}=\mathbf{f}\left(t_{i+0.5},\left([X]_{\mathrm{i}}+\frac{h}{2}[K]_{1}\right)\right)} \\
{[K]_{3}=\mathbf{f}\left(t_{i+0.5},\left([X]_{i}+\frac{h}{2}[K]_{2}\right)\right)}
\end{gathered}
$$

$$
\begin{gathered}
{[K]_{4}=\mathbf{f}\left(t_{i+1},\left([X]_{i}+h[K]_{3}\right)\right)} \\
{[K]=\frac{1}{6}\left([K]_{1}+[K]_{2}+[K]_{3}+[K]_{4}\right)}
\end{gathered}
$$

\subsection{Special Cases of $i_{q}$ and $u_{q}$}

When PWM $=0$, the armature winding is actually shortcircuited in the model, therefore, the calculated current $i_{q}$ can be of a negative value. In this case, the result should be checked. If and only if the generating EMF $e_{q}$ is greater than $u_{d c}$, where $e_{q}=\omega \Psi_{d}$, this means the armature current flows through the diode beside the IGBT and charges the power supply, therefore, $i_{q}<0$ is acceptable, and $u_{q}=u_{d c}$ should be set. Otherwise, $i_{q}=0$ and $u_{q}=e_{q}=\omega \Psi_{d}$ should be set in the calculation.

\section{PWM Control Method and Analysis}

If a PWM driving signal is applied to control the status of the IGBT, then the speed of the DC servomotor is under control. Therefore, the control strategy of the motor speed is actually reflected by the means of determining the PWM signal. The whole control system with dual closed-loops is shown in Fig. 3.

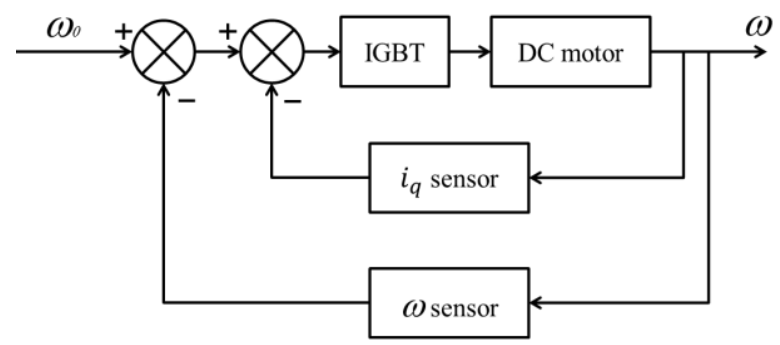

Fig. 3. Control Diagram

\subsection{Generation of PWM Signal}

There are two state values which can be obtained as feedback, the armature current $i_{q}$ and angular velocity . The aim of the control is to determine when to chop off the voltage input. One of the common methods is the dual closed-loops [6] PWM control with the armature current $i_{q}$ as the inner-loop and the angular velocity $\omega$ as the outerloop. $i_{q}$ is set to limit the current to keep the servomotor safe, and $\omega$ is the aim of the control. So the boolean status of the two loops are shown in the Table 1.

If PWM $=1$, then $u_{q}=u_{d c}$, otherwise, $u_{q}=0$. Of course the special cases discussed in subsection-2.5 should be also considered. 
Table 1. PWM values

\begin{tabular}{|c|c|c|c|}
\hline \multicolumn{2}{|c|}{$\omega$} & $\omega>\omega_{0}$ & $\omega<\omega_{0}$ \\
\cline { 3 - 4 }$i_{q}$ & 0 & 1 \\
\hline$i_{q}>$ limit & 0 & 0 & 0 \\
\hline$i_{q}<$ limit & 1 & 0 & 1 \\
\hline
\end{tabular}

\subsection{Results and Analysis}

If the aim of the velocity is set to be a square wave step between $80 \mathrm{rad} / \mathrm{s}$ and $120 \mathrm{rad} / \mathrm{s}$ every 0.2 seconds. The result is shown in Fig. 4.

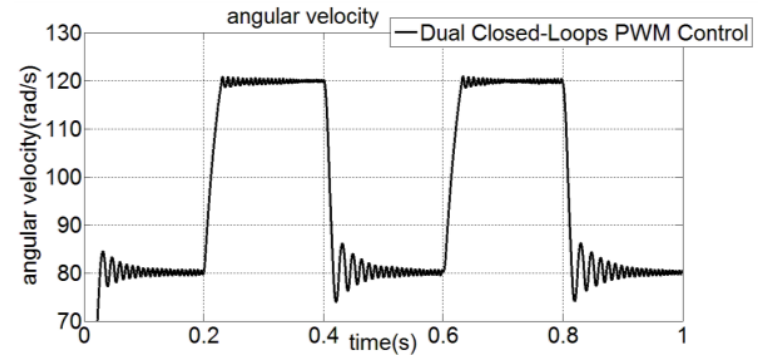

Fig. 4. Dual Closed-Loops PWM Control

It is clear that there is oscillation in the velocity control. Under Laplace transformation, differential equations (10) and (11) are modified to the following:

$$
\begin{gathered}
s \cdot i_{q}(s)=\frac{u_{q}(s)-\omega(s)-0.5 i_{q}(s)}{0.05} \\
s \cdot \omega(s)=\frac{i_{q}(s)-0.1 \omega(s)}{0.002}
\end{gathered}
$$

The relationship between $\omega$ and $u_{q}$ is

$$
\omega(s)=\frac{20}{0.002 s^{2}+0.12 s+21} u_{q}(s)
$$

It is a second-order system. According to the classical control theory, there will be overshoot and oscillation in the step response of an underdamping second-order system if it is not over-damped. Therefore, overshoot and oscillation can't be always avoided. So new control method should be found.

\subsection{Improved Dual Closed-loops Control Method}

In order to reduce overshoot and oscillation, a new control method should be found. Different researches found different control logic [7]. Notice that the relationship between $\omega$ and $i_{q}$ is

$$
\omega(s)=\frac{500}{s+50} i_{q}(s)
$$

This indicates that if $i_{q}$ is chosen to be the input of the system, then the system will turn to a first-order system, which has no overshoot or oscillation. However the response rate goes down as well.

To keep the response rapidness of the system and to reduce the overshoot and oscillation, the control method is to change the order of the system at a proper time. Thus add armature current $i_{q}$ as an inner-loop inside the loop of $\omega$. When the error of $\omega$ is small enough, the $i_{q}$ inner-loop will work.

\subsection{Improved Results and Analysis}

Under the former control settings, the results of the simulation are shown and compared in Fig. 5.

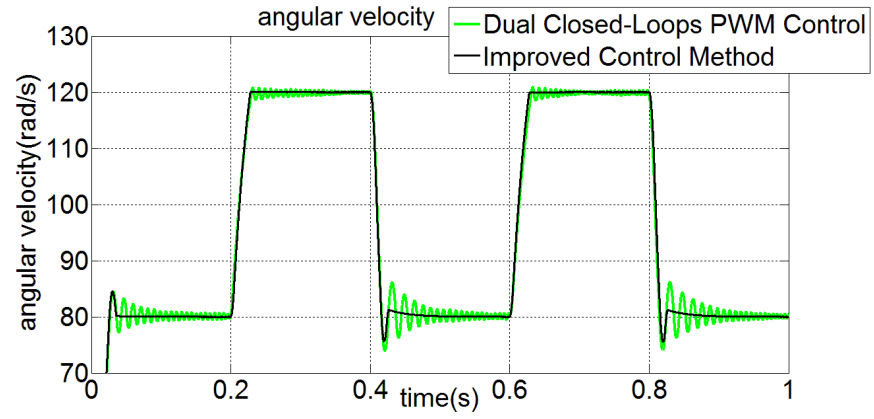

Fig. 5. Improved Control Method

There is a little overshoot at $80 \mathrm{rad} / \mathrm{s}$ but no oscillation in the improved control method. It is because the system at that time is a slightly under-damping second-order system. The overshoot is small, but the oscillation is removed, and the response is still fast. As for the oscillation at 120 rad/s, the performance is even better.

Of course, other control strategies, such as the traditional PI or PID control, and the fuzzy logic control, can all be implemented in this system. This will make the students have a clear understand of the various control methods.

\section{Conclusion}

A case study of electrical engineering education is proposed in this paper. In the study, the PM DC motor is driven with a buck power converter, whilst the motor and the power converter are both modeled with differential state equations. Various control strategies can be realized with different means of determining the buck PWM signal. The system performance is simulated by digitally solving the state equations with the fourth-order Ronge-Kutta method. In conclusion, such a project can help the undergraduate students in electrical engineering to better understand the 
electrical machines and control theories, the power electronics technology, as well as the digital simulation methodology.

\section{Acknowledgements}

The author is grateful to the supervision of his teacher, Prof. Jian-Xin Shen, and to the support of the Course Foundation of the Outstanding Engineers Program, College of Electrical Engineering, Zhejiang University.

\section{References}

[1] A. K. Pal and R. K. Mudi, "Speed control of DC motor using relay feedback tuned PI, fuzzy PI and self-tuned fuzzy PI controller," Control Theory and Informatics, vol. 2, no. 1, pp. 24-32, 2012.

[2] D. Zhao, C. Li and J. Ren, "Fuzzy speed control and stability analysis of a networked induction motor system with time delays and packet dropouts," Nonlinear Analysis: Real World Applications, vol. 12, no. 1, pp. 273-287, 2011.

[3] S. K. Gupta and P. Varshney, "Fractional fuzzy PID controller for speed control of DC motor," Proceeding in Third International Conference on Advances in Computing and Communications (ICACC 2013), pp. 1-4, 2013.

[4] R. Prakash and R. Vasanthi, "Speed control of DC-DC converter fed DC motor using robust adaptive intelligent controller," Journal of Vibration and Control, 2014.

[5] S. K. Patel, D. Sahu, V. S. Thakur, et al, "Efficient harmonics reduction based three phase H-bridge speed controller for DC motor speed control using hysteresis controlled synchronized pulse generator," International Journal of Advanced Research in Electrical and Electronics, vol. 2, no. 6, pp. 2185-2190, 2013.

[6] X. Wang, Z. Sun, L. Wang, et al, "Simulation and optimization of parameters on DC motor double closed-loop control system based on simulink," Proceeding in International Conference on Intelligent Human-Machine Systems and Cybernetics (IHMSC'09), vol. 1, pp. 153-155, 2009.

[7] P. Amiri and M. Bagheri, "Speed control of DC motor by programmable logic control with high accuracy," 2013.

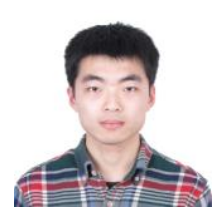

Hongtai Cao is working towards a B.S degree in electrical engineering at Zhejiang University. His research interests are smart grid systems and electric machines. 